\title{
On Gronwall type inequalities for interval-valued functions on time scales
}

\section{Awais Younus*, Muhammad Asif and Khurram Farhad}

\author{
"Correspondence: \\ awais@bzu.edu.pk \\ Centre for Advanced Studies in Pure \\ and Applied Mathematics, \\ Bahauddin Zakaryia University, \\ Multan, Pakistan
}

\begin{abstract}
In this paper, by using the concept of gH-differentiability and the Kulisch-Miranker order on intervals, we investigate some Gronwall type inequalities for interval-valued functions defined on time scales, which provide explicit bounds on unknown functions. Our results unify and extend some continuous inequalities and for discrete case all are new.
\end{abstract}

MSC: 26D15; 26E25; 39A12; 34N05

Keywords: interval-valued functions; generalized Hukuhara difference; the Kulisch-Miranker order; time scales; dynamic inequality; Gronwall inequality

\section{Introduction}

In 1919 Gronwall [1] proved that if $p$ and $f$ are real-valued continuous functions defined on $J$, where $J$ is an interval in $\mathbb{R}, t_{0} \in J$, and $f$ is differentiable in the interior $J^{0}$ of $J$, then

$$
\frac{d}{d t} f(t) \leq p(t) f(t) \quad \text { for } t \in J^{0}
$$

implies

$$
f(t) \leq f\left(t_{0}\right) \exp \left(\int_{t_{0}}^{t} p(s) d s\right) \text { for } t \in J .
$$

In 1943, Bellman [2] generalized the results as follows: if

$$
f(t) \leq q(t)+\int_{t_{0}}^{t} p(s) f(s) d s \quad \text { for } t \in J
$$

then

$$
f(t) \leq q(t)+\int_{t_{0}}^{t} q(s) p(s) \exp \left(\int_{s}^{t} p(\tau) d \tau\right) d s \quad \text { for } t \in J,
$$

where $J$ is an interval in $\mathbb{R}, t_{0} \in J$, and $f, p, q \in C\left(J, \mathbb{R}^{+}\right)$. If in addition $q(t)$ is nondecreasing, then the last inequality becomes

$$
f(t) \leq q(t) \exp \left(\int_{t_{0}}^{t} p(s) d s\right) \text { for } t \in J
$$

(c) 2015 Younus et al. This article is distributed under the terms of the Creative Commons Attribution 4.0 International License (http://creativecommons.org/licenses/by/4.0/), which permits unrestricted use, distribution, and reproduction in any medium, provided you give appropriate credit to the original author(s) and the source, provide a link to the Creative Commons license, and indicate if changes were made. 
Since the discovery of these inequalities much work has been done, and many papers which deal with new proofs, various generalizations and extensions have appeared in the literature. The above Gronwall-Bellman type inequalities are important tools to obtain various estimates in the theory of differential equations; see e.g. [3-5] and [6].

There are several mathematical models to study the behavior of the real-world systems such as: static or dynamic, linear or nonlinear, continuous or discrete, deterministic or probabilistic. In many cases the knowledge of the parameters of a real-world system is imprecise or uncertain because, generally, we cannot observe or measure with accuracy these parameters. In these situations, the parameters cannot be represented by real numbers. This shortcoming is overcome using fuzzy or interval models. Interval analysis is based on the representation of an uncertain variable as an interval of real numbers.

Interval analysis is a particular case and it has relevant applications in the treatment of the uncertainty that appears in the modeling of some real-world problems [7]. In this direction, recently several classical integral inequalities have been extended to the intervalvalued context, for instance by using the concept of gH-differentiability the authors in [8] shows an Ostrowski's inequality for interval-valued functions.

It is well known that the dynamic inequalities play important roles in the development of the qualitative theory of dynamic equations on time scales. The study of dynamic equations on time scales which goes back to its founder Hilger [9] has become an area of mathematics and recently has received a lot of attention. Recently, Luplescu in [10] developed a calculus for interval-valued functions on time scales, using the concept of a generalized Hukuhara difference provided by Markov [11].

In this paper, we investigate some Gronwall type inequalities for interval-valued functions on time scales, which generalize some inequalities from $[12,13]$ and extend some Gronwall inequalities for interval-valued functions [14] for interval-valued functions on time scales.

\section{Preliminaries}

In what follows, we recall some notions about the time scale analysis. An extensive study of the analysis on time scales can be found in [15]. Also, we outline some recent and necessary notions about differentiation of interval-valued functions. A time scale, denoted by $\mathbb{T}$, is an arbitrary, non-empty closed subset of real numbers. The operator $\sigma: \mathbb{T} \rightarrow \mathbb{T}$ called the forward jump operator is defined by $\sigma(t):=\inf \{s \in \mathbb{T}, s>t\}$. The step size function $\mu: \mathbb{T} \rightarrow \mathbb{R}_{+}$is given by $\mu(t):=\sigma(t)-t$. We say a point $t \in \mathbb{T}$ is right dense if $\mu(t)=0$, and right scattered if $\mu(t)>0$. Furthermore, a point $t \in \mathbb{T}$ is said to be left dense if $\rho(t):=$ $\sup \{s \in \mathbb{T}, s<t\}=t$ and left scattered if $\rho(t)<t$. If $\mathbb{T}$ has a left-scattered maximum $M$, then $\mathbb{T}^{k}=\mathbb{T}-\{M\}$; otherwise set $\mathbb{T}^{k}=\mathbb{T}$. Moreover, the delta derivative of a function $f: \mathbb{T} \rightarrow \mathbb{R}$ at a point $t \in \mathbb{T}^{k}$ is defined by

$$
f^{\Delta}(t)=\lim _{\substack{s \rightarrow t \\ s \neq \sigma(t)}} \frac{f(\sigma(t))-f(s)}{\sigma(t)-s} .
$$

A function $f$ is called rd-continuous provided that it is continuous at right-dense points in $\mathbb{T}$, and has finite limit at left-dense points, and the set of rd-continuous functions are denoted by $C_{\mathrm{rd}}(\mathbb{T}, \mathbb{R})$. The set of functions $C_{\mathrm{rd}}^{1}(\mathbb{T}, \mathbb{R})$ includes the functions $f$ whose derivative is in $C_{\mathrm{rd}}(\mathbb{T}, \mathbb{R})$ too. For $s, t \in \mathbb{T}$ and a function $f \in C_{\mathrm{rd}}(\mathbb{T}, \mathbb{R})$, the $\Delta$-integral is defined 
to be

$$
\int_{s}^{t} f(\tau) \Delta \tau=F(t)-F(s),
$$

where $F \in C_{\mathrm{rd}}^{1}(\mathbb{T}, \mathbb{R})$ is an anti-derivative of $f$, i.e., $F^{\Delta}=f$ on $\mathbb{T}^{k}$.

A function $f \in C_{\mathrm{rd}}(\mathbb{T}, \mathbb{R})$ is called regressive if $1+\mu(t) f(t) \neq 0$ for all $t \in \mathbb{T}^{k}$, and $f \in$ $C_{\mathrm{rd}}(\mathbb{T}, \mathbb{R})$ is called positively regressive if $1+\mu(t) f(t)>0$ on $\mathbb{T}^{k}$. The set of regressive functions and the set of positively regressive functions are denoted by $\mathcal{R}(\mathbb{T}, \mathbb{R})$ and $\mathcal{R}^{+}(\mathbb{T}, \mathbb{R})$, respectively.

Let $f \in \mathcal{R}(\mathbb{T}, \mathbb{R})$ and $s \in \mathbb{T}$, then the generalized exponential function $e_{f}(\cdot, s)$ on a time scale $\mathbb{T}$ is defined to be the unique solution of the following initial value problem:

$$
\left\{\begin{array}{l}
x^{\Delta}(t)=f(t) x(t) \\
x(s)=1
\end{array}\right.
$$

For $h \in \mathbb{R}^{+}$, set $\mathbb{C}_{h}:=\{z \in \mathbb{C}: z \neq-1 / h\}, \mathbb{Z}_{h}:=\{z \in \mathbb{C}:-\pi / h<\operatorname{Im}(z) \leq \pi / h\}$, and $\mathbb{C}_{0}:=\mathbb{Z}_{0}:=\mathbb{C}$. For $h \in \mathbb{R}_{0}^{+}$and $z \in \mathbb{C}_{h}$, the cylinder transformation $\xi_{h}: \mathbb{C}_{h} \rightarrow \mathbb{Z}_{h}$ is defined by

$$
\xi_{h}(z):= \begin{cases}z, & h=0 \\ \frac{1}{h} \log (1+z h), & h>0\end{cases}
$$

and the exponential function can also be written in the form

$$
e_{f}(t, s):=\exp \left\{\int_{s}^{t} \xi_{\mu(\tau)}(f(\tau)) \Delta \tau\right\} \quad \text { for } s, t \in \mathbb{T} \text {. }
$$

For further details as regards these notions one may consult [15].

Let $\mathcal{K}_{C}$ be the set of all non-empty compact intervals of the real line $\mathbb{R}$. Also $\mathcal{K}_{C}^{+}=$ $\left\{[a, b] \in \mathcal{K}_{C}: 0 \leq a \leq b\right\}$. If $\left[a^{-}, a^{+}\right],\left[b^{-}, b^{+}\right] \in \mathcal{K}_{C}$, then the usual interval operations, i.e., Minkowski addition and scalar multiplication, are defined by

$$
\left[a^{-}, a^{+}\right]+\left[b^{-}, b^{+}\right]=\left[a^{-}+b^{-}, a^{+}+b^{+}\right]
$$

and

$$
\lambda\left[a^{-}, a^{+}\right]= \begin{cases}{\left[\lambda a^{-}, \lambda a^{+}\right]} & \text {if } \lambda>0, \\ \{0\} & \text { if } \lambda=0, \\ {\left[\lambda a^{+}, \lambda a^{-}\right]} & \text {if } \lambda<0,\end{cases}
$$

respectively. If $\lambda=-1$, then $(-1)\left[a^{-}, a^{+}\right]=-\left[a^{-}, a^{+}\right]=\left[-a^{+},-a^{-}\right]$.

Also, the Kulisch-Miranker order for $\left[a^{-}, a^{+}\right],\left[b^{-}, b^{+}\right] \in \mathcal{K}_{C}$ is defined as follows [16]:

$$
\left[a^{-}, a^{+}\right] \leq\left[b^{-}, b^{+}\right] \quad \text { if and only if } a^{-} \leq b^{-} \text {and } a^{+} \leq b^{+} \text {. }
$$

For two intervals $\left[a^{-}, a^{+}\right],\left[b^{-}, b^{+}\right] \in \mathcal{K}_{C}$, the generalized Hukuhara difference (gHdifference for short) is defined as follows $[11,17]$ :

$$
\left[a^{-}, a^{+}\right] \ominus_{g}\left[b^{-}, b^{+}\right]=\left[\min \left\{a^{-}-b^{-}, a^{+}-b^{+}\right\}, \max \left\{a^{-}-b^{-}, a^{+}-b^{+}\right\}\right] .
$$


For $A=\left[a^{-}, a^{+}\right] \in \mathcal{K}_{C}$, len $(A)=a^{+}-a^{-}$is called the length of interval $A$. Then, for $A=$ $\left[a^{-}, a^{+}\right]$and $B=\left[b^{-}, b^{+}\right]$, we have

$$
A \ominus_{g} B= \begin{cases}{\left[a^{-}-b^{-}, a^{+}-b^{+}\right]} & \text {if } \operatorname{len}(A) \geq \operatorname{len}(B), \\ {\left[a^{+}-b^{+}, a^{-}-b^{-}\right]} & \text {if } \operatorname{len}(A)<\operatorname{len}(B) .\end{cases}
$$

If $A, B, C \in \mathcal{K}_{C}$ then

$$
A \ominus_{g} B=C \Longleftrightarrow \begin{cases}A=B+C & \text { if } \operatorname{len}(A) \geq \operatorname{len}(B), \\ B=A+(-C) & \text { if } \operatorname{len}(A)<\operatorname{len}(B) .\end{cases}
$$

A metric structure, the Hausdorff-Pompeiu distance $D: \mathcal{K}_{C} \times \mathcal{K}_{C} \rightarrow[0, \infty)$ defined by $D(A, B)=\max \left\{\left|a^{-}-b^{-}\right|,\left|a^{+}-b^{+}\right|\right\}$, where $A=\left[a^{-}, a^{+}\right]$and $B=\left[b^{-}, b^{+}\right]$, is given, satisfying

$$
\begin{array}{ll}
\left(\mathrm{D}_{1}\right) & D(A, B)=0 \quad \Longleftrightarrow \quad A=B, \\
\left(\mathrm{D}_{2}\right) & D(\lambda A, \lambda B)=|\lambda| D(A, B) \quad \text { for all } \lambda \in \mathbb{R}, \\
\left(\mathrm{D}_{3}\right) & D(A+C, B+C)=D(A, B), \\
\left(\mathrm{D}_{4}\right) & D(A+B, C+D) \leq D(A, C)+D(B, D), \\
\left(\mathrm{D}_{5}\right) & D(A, B)=D\left(A \ominus_{g} B,\{0\}\right) .
\end{array}
$$

Also, $\left(\mathcal{K}_{C}, D\right)$ is a complete and separable metric space. Moreover, the limits and continuity can be characterized, in the metric space $\left(\mathcal{K}_{C}, D\right)$, by the gH-difference.

Next we recall the basics of the calculus of interval-valued functions on time scales (see [10]).

An interval-valued function $F: \mathbb{T} \rightarrow \mathcal{K}_{C}$ has a $\mathbb{T}$-limit $A \in \mathcal{K}_{C}$ at $t_{0} \in \mathbb{T}$ if for every $\varepsilon>0$, there exists $\delta>0$ such that $D\left(F(t) \ominus_{g} A,\{0\}\right) \leq \varepsilon$ for all $t \in U_{\mathbb{T}}\left(t_{0}, \delta\right)$. If $F$ has a $\mathbb{T}$-limit $A \in \mathcal{K}_{C}$ at $t_{0} \in \mathbb{T}$, then it is unique and is denoted by $\mathbb{T}$ - $\lim _{t \rightarrow t_{0}} F(t)$. An intervalvalued function $F: \mathbb{T} \rightarrow \mathcal{K}_{C}$ is continuous at $t_{0} \in \mathbb{T}$, if $\mathbb{T}-\lim _{t \rightarrow t_{0}} F(t) \in \mathcal{K}_{C}$ exists and $\mathbb{T}-\lim _{t \rightarrow t_{0}} F(t)=F\left(t_{0}\right)$, that is, $\mathbb{T}-\lim _{t \rightarrow t_{0}} F(t) \ominus_{g} F\left(t_{0}\right)=\{0\}$. An interval-valued function $F: \mathbb{T} \rightarrow \mathcal{K}_{C}$ is called regulated if its right-sided $\mathbb{T}$-limits (in $\mathcal{K}_{C}$ ) exist at all right-dense points in $\mathbb{T}$, and its left-sided $\mathbb{T}$-limits (in $\mathcal{K}_{C}$ ) exist at all left-dense points in $\mathbb{T}$. An intervalvalued function $F: \mathbb{T} \rightarrow \mathcal{K}_{C}$ is called $r d$-continuous if it is continuous at all right-dense points in $\mathbb{T}$ and its left-sided $\mathbb{T}$-limits (in $\mathcal{K}_{C}$ ) exist at all left-dense points in $\mathbb{T}$.

It is quite obvious that if $F: \mathbb{T} \rightarrow \mathcal{K}_{C}$, such that $F(t)=\left[f^{-}(t), f^{+}(t)\right]$, then $F$ is continuous (regulated, rd-continuous) if and only if $f^{-}$and $f^{+}$are continuous (regulated, rd-continuous). It follows that a continuous interval-valued function is rd-continuous, and a rd-continuous interval-valued function is regulated.

Let $F: \mathbb{T} \rightarrow \mathcal{K}_{C}$ and $t \in \mathbb{T}^{\kappa}$. We define $F^{\Delta}(t) \in \mathcal{K}_{C}$ (provided it exists) with the property that for every $\varepsilon>0$, there is $\delta>0$ such that

$$
D\left(F(\sigma(t)) \ominus_{g} F(s),[\sigma(t)-s] F^{\Delta}(t)\right) \leq \varepsilon|\sigma(t)-s|
$$

for all $s \in U_{\mathbb{T}}(t, \delta)$. We call $F^{\Delta}(t)$ the delta generalized Hukuhara derivative $\left(\Delta_{\mathrm{gH}}\right.$-derivative for short) of $F$ at $t$. Moreover, we say that $F$ is delta generalized Hukuhara differentiable 
( $\Delta_{\mathrm{gH}}$-differentiable for short) on $\mathbb{T}^{\kappa}$ if $F^{\Delta}(t) \in \mathcal{K}_{C}$ exists at each point $t \in \mathbb{T}^{\kappa}$. The intervalvalued function $F^{\Delta}: \mathbb{T}^{\kappa} \rightarrow \mathcal{K}_{C}$ is called the $\Delta_{\mathrm{gH}}$ derivative of $F$ on $\mathbb{T}^{\kappa}$.

Obviously, if $F: \mathbb{T} \rightarrow \mathcal{K}_{C}$ is a constant interval-valued function given by $F(t)=A \in \mathcal{K}_{C}$, then $F^{\Delta}(t)=\{0\}$ for all $t \in \mathbb{T}^{\kappa}$.

Theorem 2.1 ([10]) Assume that $F: \mathbb{T} \rightarrow \mathcal{K}_{C}$ is a given interval-valued function and let $t \in \mathbb{T}^{\kappa}$. Then we have the following:

(i) If $F: \mathbb{T} \rightarrow \mathcal{K}_{C}$ is $\Delta_{\mathrm{gH}^{-}}$-differentiable at $t \in \mathbb{T}^{\kappa}$ then it is continuous at $t$.

(ii) If $F$ is continuous at $t$ and $t$ is right scattered, then $F$ is $\Delta_{\mathrm{gH}}$-differentiable at $t$ with

$$
F^{\Delta}(t)=\frac{F(\sigma(t)) \ominus_{g} F(t)}{\mu(t)} .
$$

(iii) If $t$ is right dense, then $F$ is $\Delta_{\mathrm{gH}^{-}}$-differentiable at $t$ if and only if the $\mathbb{T}$-limit

$$
\mathbb{T}-\lim _{s \rightarrow t} \frac{F(t) \ominus_{g} F(s)}{t-s}
$$

exists. In this case

$$
F^{\Delta}(t)=\mathbb{T}-\lim _{s \rightarrow t} \frac{F(t) \ominus_{g} F(s)}{t-s} .
$$

(iv) If $F$ is $\Delta_{\mathrm{gH}^{-}}$-differentiable at $t$, then

$$
F(\sigma(t)) \ominus_{g} F(t)=\mu(t) F^{\Delta}(t)
$$

Theorem 2.2 Let $F: \mathbb{T} \rightarrow \mathcal{K}_{C}$ be such that $F(t)=\left[f^{-}(t), f^{+}(t)\right], t \in \mathbb{T}$. If the real-valued functions $f^{-}$and $f^{+}$are $\Delta$-differentiable at $t_{0} \in \mathbb{T}^{\kappa}$, then $F$ is $\Delta_{\mathrm{gH}}$-differentiable at $t_{0} \in \mathbb{T}^{\kappa}$ and

$$
F^{\Delta}(t)=\left[\min \left\{\left(f^{-}\right)^{\Delta}\left(t_{0}\right),\left(f^{+}\right)^{\Delta}\left(t_{0}\right)\right\}, \max \left\{\left(f^{-}\right)^{\Delta}\left(t_{0}\right),\left(f^{+}\right)^{\Delta}\left(t_{0}\right)\right\}\right]
$$

Remark 2.3 The converse of Theorem 2.2 does not true, that is, the $\Delta_{\mathrm{gH}}$-differentiability of $F$ does not imply the $\Delta$-differentiability of $f^{-}$and $f^{+}$(see $[11,18]$ ).

As a consequence of Theorem 2.2, we have, if $g: \mathbb{T} \rightarrow \mathbb{R}$ be a $\Delta$-differentiable at $t_{0} \in \mathbb{T}^{\kappa}$, $C=[a, b], a<b$, be a constant interval and $F: \mathbb{T} \rightarrow \mathcal{K}_{C}$ be an interval-valued function given by $F(t)=C g(t)$. Then $F$ is $\Delta_{\mathrm{gH}}$-differentiable at $t_{0} \in \mathbb{T}^{\kappa}$ and $F^{\Delta}(t)=C g^{\Delta}(t)$.

Definition 2.4 An interval-valued function $F: \mathbb{T} \rightarrow \mathcal{K}_{C}$ is l-nondecreasing (l-nonincreasing) on $\mathbb{T}$ if the real function $t \mapsto \operatorname{len}(F(t))$ nondecreasing (nonincreasing) on $\mathbb{T}$. If $F$ is nondecreasing or nonincreasing on $\mathbb{T}$, then we say that $F$ is $l$-monotone on $\mathbb{T}$.

Theorem 2.5 Let $F:[a, b]_{\mathbb{T}} \rightarrow \mathcal{K}_{C}$ be such that $F(t)=\left[f^{-}(t), f^{+}(t)\right], t \in[a, b]_{\mathbb{T}}$. If $F$ is l-monotone on $[a, b]_{\mathbb{T}}$ and $\Delta_{\mathrm{gH}}$-differentiable on $[a, b)_{\mathbb{T}}$, then $\left(f^{-}\right)^{\Delta}(t)$ and $\left(f^{+}\right)^{\Delta}(t)$ exist for all $t \in[a, b)_{\mathbb{T}}$. Moreover, we have:

(i) $F^{\Delta}(t)=\left[\left(f^{-}\right)^{\Delta}(t),\left(f^{+}\right)^{\Delta}(t)\right]$ for all $t \in[a, b)_{\mathbb{T}}$, if $F$ is l-nondecreasing;

(ii) $F^{\Delta}(t)=\left[\left(f^{+}\right)^{\Delta}(t),\left(f^{-}\right)^{\Delta}(t)\right]$ for all $t \in[a, b)_{\mathbb{T}}$, if $F$ is l-nonincreasing. 
Theorem 2.6 Let $F:[a, b]_{\mathbb{T}} \rightarrow \mathcal{K}_{C}$ be l-monotone on $[a, b]_{\mathbb{T}}$ and $\Delta_{\mathrm{gH}^{-}}$-differentiable on $[a, b)_{\mathbb{T}}$. We say that $F$ is $\Delta_{1, \mathrm{gH}}$-differentiable (or first type Hukuhara differentiable [19]) on $[a, b)_{\mathbb{T}}$ if

(i) $F^{\Delta}(t)=\left[\left(f^{-}\right)^{\Delta}(t),\left(f^{+}\right)^{\Delta}(t)\right]$ for all $t \in[a, b)_{\mathbb{T}}$,

and $\Delta_{2, \mathrm{gH}^{-}}$differentiable (or second type Hukuhara differentiable [19]) on $[a, b)_{\mathbb{T}}$ if

(ii) $F^{\Delta}(t)=\left[\left(f^{+}\right)^{\Delta}(t),\left(f^{-}\right)^{\Delta}(t)\right]$ for all $t \in[a, b)_{\mathbb{T}}$.

Let $F: \mathbb{T} \rightarrow \mathcal{K}_{C}$ be an interval-valued function and let $P: a=t_{0}<t_{1}<\cdots<t_{n}=b$ be a partition of $[a, b)_{\mathbb{T}}$. In each interval $\left[t_{i-1}, t_{i}\right)_{\mathbb{T}}$, where $1 \leq i \leq n$, choose an arbitrary point $\xi_{i}$ and form the sum

$$
S=\sum_{i=1}^{n}\left(t_{i}-t_{i-1}\right) F\left(\xi_{i}\right)
$$

We call $S$ a Riemann $\Delta$-sum of $F$ corresponding to the partition $P$.

A bounded interval-valued function $F: \mathbb{T} \rightarrow \mathcal{K}_{C}$ is Riemann $\Delta$-integrable from $a$ to $b$ (or on $[a, b)_{\mathbb{T}}$ ) if there is $A \in \mathcal{K}_{C}$ such that for each $\varepsilon>0$ there is $\delta>0$ such that

$$
D\left(S \ominus_{g} A,\{0\}\right)<\varepsilon
$$

for every Riemann $\Delta$-sum $S$ of $F$ corresponding to a partition $P \in \mathcal{P}\left([a, b)_{\mathbb{T}}, \delta\right)$ independent of the way in which we choose $\xi_{i} \in\left[t_{i-1}, t_{i}\right)_{\mathbb{T}}, i=1,2, \ldots, n$. It is easily seen that $A \in \mathcal{K}_{C}$ is unique. $A \in \mathcal{K}_{C}$ is called the Riemann $\Delta$-integral of $F$ from $a$ to $b$, and we will denote it by $\int_{a}^{b} F(t) \Delta t$.

Theorem 2.7 Let $F: \mathbb{T} \rightarrow \mathcal{K}_{C}$ be an interval-valued function such that $F(t)=\left[f^{-}(t), f^{+}(t)\right]$. Then $F$ is Riemann $\Delta$-integrable on $[a, b)_{\mathbb{T}}$ if and only if $f^{-}$and $f^{+}$are Riemann $\Delta$-integrable on $[a, b)_{\mathbb{T}}$. Moreover, we have

$$
\int_{a}^{b} F(t) \Delta t=\left[\int_{a}^{b} f^{-}(t) \Delta t, \int_{a}^{b} f^{+}(t) \Delta t\right]
$$

Proposition 2.8 Assume that $a, b \in \mathbb{T}, a<b$ and $F: \mathbb{T} \rightarrow \mathcal{K}_{C}$ is $r d$-continuous. Then the integral has the following properties:

(i) If $\mathbb{T}=\mathbb{R}$, then $\int_{a}^{b} F(t) \Delta t=\int_{a}^{b} F(t) d t=\left[\int_{a}^{b} f^{-}(t) d t, \int_{a}^{b} f^{+}(t) d t\right]$, where the integral on the right-hand side is the Riemann integral.

(ii) If $\mathbb{T}$ consists of isolated points, then

$$
\int_{a}^{b} F(t) \Delta t=\sum_{t \in[a, b)_{\mathbb{T}}} \mu(t) F(t)=\left[\sum_{t \in[a, b)_{\mathbb{T}}} \mu(t) f^{-}(t), \sum_{t \in[a, b)_{\mathbb{T}}} \mu(t) f^{+}(t)\right] .
$$

\section{Gronwall type inequalities}

Theorem 3.1 Let $F, Y \in C_{\mathrm{rd}}\left([a, b]_{\mathbb{T}}, \mathcal{K}_{C}\right)$ and $p \in \mathcal{R}^{+}\left([a, b]_{\mathbb{T}}, \mathbb{R}\right)$. 
(a) If $p(t) \geq 0$ on $[a, b]_{\mathbb{T}}, Y$ is $\Delta_{1, \mathrm{gH}}$-differentiable on $[a, b)_{\mathbb{T}}$ and satisfies the interval dynamic inequality

$$
Y^{\Delta}(t) \leq p(t) Y(t)+F(t) \quad \text { for all } t \in[a, b)_{\mathbb{T}},
$$

then

$$
Y(t) \leq e_{p}(t, a) Y(a)+\int_{a}^{t} e_{p}(t, \sigma(\tau)) F(t) \Delta \tau
$$

for all $t \in[a, b]_{\mathbb{T}}$.

(b) If $p(t) \leq 0$ on $[a, b]_{\mathbb{T}}, Y$ is $\Delta_{2, \mathrm{gH}^{-}}$differentiable on $[a, b)_{\mathbb{T}}$ and satisfies the interval dynamic inequality, (9) then (10) holds, for all $t \in[a, b]_{\mathbb{T}}$.

Proof Let $Y, F:[a, b]_{\mathbb{T}} \rightarrow \mathcal{K}_{C}$ be interval-valued functions such that $Y(t)=\left[y^{-}(t), y^{+}(t)\right]$ and $F(t)=\left[f^{-}(t), f^{+}(t)\right]$ and both are rd-continuous on $[a, b]_{\mathbb{T}}$.

(a) If $Y(t)$ is $\Delta_{1, \mathrm{gH}}$-differentiable, then by using Theorem $2.6 Y^{\Delta}(t)=\left[\left(y^{-}\right)^{\Delta}(t),\left(y^{+}\right)^{\Delta}(t)\right]$. Since $p(t) \geq 0$ on $[a, b]_{\mathbb{T}}$ we have $p(t) Y(t)=\left[p(t) y^{-}(t), p(t) y^{+}(t)\right]$. By using inequality (9) we obtain

$$
\left[\left(y^{-}\right)^{\Delta}(t),\left(y^{+}\right)^{\Delta}(t)\right] \leq\left[p(t) y^{-}(t)+f^{-}(t), p(t) y^{+}(t)+f^{+}(t)\right]
$$

Apply the Kulisch-Miranker order (1), we have

$$
\begin{aligned}
& \left(y^{-}\right)^{\Delta}(t) \leq p(t) y^{-}(t)+f^{-}(t), \\
& \left(y^{+}\right)^{\Delta}(t) \leq p(t) y^{+}(t)+f^{+}(t) .
\end{aligned}
$$

By using Theorem 6.1 of [15] on (11) and (12), respectively, we obtain

$$
\begin{aligned}
& y^{-}(t) \leq e_{p}(t, a) y^{-}(a)+\int_{a}^{t} e_{p}(t, \sigma(\tau)) f^{-}(t) \Delta \tau, \\
& y^{+}(t) \leq e_{p}(t, a) y^{+}(a)+\int_{a}^{t} e_{p}(t, \sigma(\tau)) f^{+}(t) \Delta \tau .
\end{aligned}
$$

Again the Kulisch-Miranker order (1) on inequalities (13) and (14) yields

$$
\begin{aligned}
Y(t)= & {\left[y^{-}(t), y^{+}(t)\right] } \\
\leq & {\left[e_{p}(t, a) y^{-}(a)+\int_{a}^{t} e_{p}(t, \sigma(\tau)) f^{-}(\tau) \Delta \tau, e_{p}(t, a) y^{+}(a)\right.} \\
& \left.+\int_{a}^{t} e_{p}(t, \sigma(\tau)) f^{+}(\tau) \Delta \tau\right] \\
= & {\left[e_{p}(t, a) y^{-}(a), e_{p}(t, a) y^{+}(a)\right] } \\
& +\left[\int_{a}^{t} e_{p}(t, \sigma(\tau)) f^{-}(\tau) \Delta \tau, \int_{a}^{t} e_{p}(t, \sigma(\tau)) f^{+}(\tau) \Delta \tau\right] \\
= & e_{p}(t, a)\left[y^{-}(a), y^{+}(a)\right]+\int_{a}^{t}\left[e_{p}(t, \sigma(\tau)) f^{-}(\tau), e_{p}(t, \sigma(\tau)) f^{+}(\tau)\right] \Delta \tau
\end{aligned}
$$




$$
\begin{aligned}
& =e_{p}(t, a) Y(a)+\int_{a}^{t} e_{p}(t, \sigma(\tau))\left[f^{-}(\tau), f^{+}(\tau)\right] \Delta \tau \\
& =e_{p}(t, a) Y(a)+\int_{a}^{t} e_{p}(t, \sigma(\tau)) F(\tau) \Delta \tau
\end{aligned}
$$

This proves part (a).

(b) If $Y(t)$ is $\Delta_{2, \mathrm{gH}}$-differentiable, then $Y^{\Delta}(t)=\left[\left(y^{+}\right)^{\Delta}(t),\left(y^{-}\right)^{\Delta}(t)\right]$ and as $p(t) \leq 0$, so we have $p(t) Y(t)=\left[p(t) y^{-}(t), p(t) y^{+}(t)\right]$ and the remaining proof follows as (a).

Corollary 3.2 Let $Y \in C_{\mathrm{rd}}\left([a, b]_{\mathbb{T}}, \mathcal{K}_{C}\right)$ and $p \in \mathcal{R}^{+}\left([a, b]_{\mathbb{T}}, \mathbb{R}\right)$.

(a) If $p(t) \geq 0$ on $[a, b]_{\mathbb{T}}, Y$ is $\Delta_{1, \mathrm{gH}}$-differentiable on $[a, b)_{\mathbb{T}}$ and satisfies the interval dynamic inequality

$$
Y^{\Delta}(t) \leq p(t) Y(t) \quad \text { for all } t \in[a, b)_{\mathbb{T}}
$$

then

$$
Y(t) \leq e_{p}(t, a) Y(a)
$$

for all $t \in[a, b]_{\mathbb{T}}$.

(b) If $p(t) \leq 0$ on $[a, b]_{\mathbb{T}}, Y$ is $\Delta_{2, \mathrm{gH}^{-}}$-differentiable on $[a, b)_{\mathbb{T}}$ and satisfies the interval dynamic inequality (15) then (16) holds, for all $t \in[a, b]_{\mathbb{T}}$.

Theorem 3.3 Let $F, Y \in C_{\mathrm{rd}}\left([a, b]_{\mathbb{T}}, \mathcal{K}_{C}\right)$ and $p \in \mathcal{R}^{+}\left([a, b]_{\mathbb{T}}, \mathbb{R}\right)$.

(a) If $p(t) \leq 0$ on $[a, b]_{\mathbb{T}}, Y$ is $\Delta_{1, \mathrm{gH}}$-differentiable on $[a, b)_{\mathbb{T}}$ and satisfies the interval dynamic inequality

$$
Y^{\Delta}(t) \leq-p(t) Y^{\sigma}(t)+F(t) \quad \text { for all } t \in[a, b)_{\mathbb{T}},
$$

then

$$
Y(t) \leq e_{\ominus p}(t, a) Y(a)+\int_{a}^{t} e_{\ominus p}(t, \tau) F(t) \Delta \tau
$$

for all $t \in[a, b]_{\mathbb{T}}$.

(b) If $p(t) \geq 0$ on $[a, b]_{\mathbb{T}}, Y$ is $\Delta_{2, \mathrm{gH}^{-}}$-differentiable on $[a, b)_{\mathbb{T}}$ and satisfies the interval dynamic inequality (17) then (18) holds, for all $t \in[a, b]_{\mathbb{T}}$.

Proof Let $F, Y:[a, b]_{\mathbb{T}} \rightarrow \mathcal{K}_{C}$ be interval-valued functions such that $Y(t)=\left[y^{-}(t), y^{+}(t)\right]$ and $F(t)=\left[f^{-}(t), f^{+}(t)\right]$ and both are rd-continuous on $[a, b]_{\mathbb{T}}$.

(a) If $Y(t)$ is $\Delta_{1, \mathrm{gH}}$-differentiable on $[a, b)_{\mathbb{T}}$, then by using Theorem $2.6 Y^{\Delta}(t)=\left[\left(y^{-}\right)^{\Delta}(t)\right.$, $\left.\left(y^{+}\right)^{\Delta}(t)\right]$. Since $p(t) \leq 0$ on $[a, b]_{\mathbb{T}}$, we have $-p(t) Y^{\sigma}(t)=\left[-p(t) y^{-}(\sigma(t)),-p(t) y^{+}(\sigma(t))\right]$. By using (17) we obtain

$$
\left[\left(y^{-}\right)^{\Delta}(t),\left(y^{+}\right)^{\Delta}(t)\right] \leq\left[-p(t) y^{-}(\sigma(t))+f^{-}(t),-p(t) y^{+}(\sigma(t))+f^{+}(t)\right] .
$$

By using the Kulisch-Miranker order (1), we have

$$
\left(y^{-}\right)^{\Delta}(t) \leq-p(t) y^{-}(\sigma(t))+f^{-}(t)
$$




$$
\left(y^{+}\right)^{\Delta}(t) \leq-p(t) y^{+}(\sigma(t))+f^{+}(t)
$$

Using Theorem 3.5 of [20] on (19) and (20), it follows that

$$
\begin{aligned}
& y^{-}(t) \leq e_{\ominus p}(t, a) y^{-}(a)+\int_{a}^{t} e_{\ominus p}(t, \tau) f^{-}(t) \Delta \tau, \\
& y^{+}(t) \leq e_{\ominus p}(t, a) y^{+}(a)+\int_{a}^{t} e_{\ominus p}(t, \tau) f^{+}(t) \Delta \tau .
\end{aligned}
$$

The Kulisch-Miranker order (1) on (21) and (22), respectively, yields

$$
\begin{aligned}
Y(t)= & {\left[y^{-}(t), y^{+}(t)\right] } \\
\leq & {\left[e_{\ominus p}(t, a) y^{-}(a)+\int_{a}^{t} e_{\ominus p}(t, \tau) f^{-}(t) \Delta \tau, e_{\ominus p}(t, a) y^{+}(a)\right.} \\
& \left.+\int_{a}^{t} e_{\ominus p}(t, \tau) f^{+}(t) \Delta \tau\right] \\
= & {\left[e_{\ominus p}(t, a) y^{-}(a), e_{\ominus p}(t, a) y^{+}(a)\right] } \\
& +\left[\int_{a}^{t} e_{\ominus p}(t, \tau) f^{-}(\tau) \Delta \tau, \int_{a}^{t} e_{\ominus p}(t, \tau) f^{+}(\tau) \Delta \tau\right] \\
= & e_{\ominus p}(t, a)\left[y^{-}(a), y^{+}(a)\right]+\int_{a}^{t}\left[e_{\ominus p}(t, \tau) f^{-}(\tau), e_{\ominus p}(t, \tau) f^{+}(\tau)\right] \Delta \tau \\
= & e_{\ominus p}(t, a) Y(a)+\int_{a}^{t} e_{\ominus p}(t, \tau)\left[f^{-}(\tau), f^{+}(\tau)\right] \Delta \tau \\
= & e_{\ominus p}(t, a) Y(a)+\int_{a}^{t} e_{\ominus p}(t, \sigma(\tau)) F(\tau) \Delta \tau .
\end{aligned}
$$

This proves part (a).

(b) If $Y(t)$ is $\Delta_{2, \mathrm{gH}}$-differentiable on $[a, b)_{\mathbb{T}}$, then by using Theorem 2.6 and as $p(t) \leq 0$ we have $p(t) Y^{\sigma}(t)=\left[p(t) y^{-}(\sigma(t)), p(t) y^{+}(\sigma(t))\right]$ and the remaining proof follows as (a).

Theorem 3.4 Let $F, Y \in C_{\mathrm{rd}}\left([a, b]_{\mathbb{T}}, \mathcal{K}_{C}\right)$ and $p \in \mathcal{R}^{+}\left([a, b]_{\mathbb{T}}, \mathbb{R}\right)$.

(a) If $p(t) \geq 0$ on $[a, b]_{\mathbb{T}}, Y$ is $\Delta_{1, \mathrm{gH}}$-differentiable on $[a, b)_{\mathbb{T}}$ and satisfies the interval dynamic inequality

$$
Y^{\Delta}(t) \leq-p(t) Y^{\sigma}(t)+F(t) \quad \text { for all } t \in[a, b]_{\mathbb{T}}
$$

then

$$
Y(t) \geq e_{\ominus p}(t, a) Y(a)+\int_{a}^{t} e_{\ominus p}(t, \sigma(\tau)) F(t) \Delta \tau
$$

for all $t \in[a, b]_{\mathbb{T}}$.

(b) If $p(t) \leq 0$ on $[a, b]_{\mathbb{T}}, Y$ is $\Delta_{2, \mathrm{gH}}$-differentiable on $[a, b)_{\mathbb{T}}$ and satisfies the interval dynamic inequality (23) then it satisfies (24).

Theorem 3.5 Let $F:[a, b]_{\mathbb{T}} \rightarrow \mathcal{K}_{C}$ be an interval-valued function given by $F(t)=X_{0} g(t)$, where $X_{0} \in \mathcal{K}_{C}^{+}$is a constant interval and $g:[a, b]_{\mathbb{T}} \rightarrow \mathbb{R}$ be a $\Delta$-differentiable on $[a, b)_{\mathbb{T}}$ 
that satisfies the dynamic inequality

$$
g^{\Delta}(t) \leq p(t) g(t)
$$

then the following hold:

(i) $\quad F^{\Delta}(t) \leq p(t) F(t)$

and

(ii) $\quad F(t) \leq F(a) e_{p}(t, a)$.

Proof (i) $g(t)$ is $\Delta$-differentiable and so is $\Delta_{\mathrm{gH}}$-differentiable and $F^{\Delta}(t)=X_{0} g^{\Delta}(t)$; by inequality (25)

$$
F^{\Delta}(t)=X_{0} g^{\Delta}(t) \leq X_{0} p(t) g(t)
$$

which implies

$$
F^{\Delta}(t) \leq p(t) F(t)
$$

(ii) Since $g(t)$ satisfies (25), by Theorem 6.1 of [15], we have

$$
g(t) \leq g(a) e_{p}(t, a) .
$$

It follows that

$$
F(t)=X_{0} g(t) \leq X_{0} g(a) e_{p}(t, a)
$$

and we have

$$
F(t) \leq F(a) e_{p}(t, a)
$$

Theorem 3.6 Let $F, Y \in C_{\mathrm{rd}}\left([a, b]_{\mathbb{T}}, \mathcal{K}_{C}\right)$ and $p \in \mathcal{R}^{+}\left([a, b]_{\mathbb{T}}, \mathbb{R}\right), p(t) \geq 0$ for all $t \in[a, b]_{\mathbb{T}}$, such that the interval integral inequality

$$
Y(t) \leq F(t)+\int_{a}^{t} Y(\tau) p(\tau) \Delta \tau
$$

holds for all $t \in[a, b]_{\mathbb{T}}$. Then

$$
Y(t) \leq F(t)+\int_{a}^{t} e_{p}(t, \sigma(\tau)) F(\tau) p(\tau) \Delta \tau
$$

for all $t \in[a, b]_{\mathbb{T}}$.

Proof Let $F, Y:[a, b]_{\mathbb{T}} \rightarrow \mathcal{K}_{C}$ be interval-valued functions such that $Y(t)=\left[y^{-}(t), y^{+}(t)\right]$ and $F(t)=\left[f^{-}(t), f^{+}(t)\right]$. Then from inequality (26) we obtain

$$
\left[y^{-}(t), y^{+}(t)\right] \leq\left[f^{-}(t)+\int_{a}^{t} y^{-}(\tau) p(\tau) \Delta \tau, f^{+}(t)+\int_{a}^{t} y^{+}(\tau) p(\tau) \Delta \tau\right] .
$$


The Kulisch-Miranker order (1) yields

$$
\begin{aligned}
& y^{-}(t) \leq f^{-}(t)+\int_{a}^{t} y^{-}(\tau) p(\tau) \Delta \tau \\
& y^{+}(t) \leq f^{+}(t)+\int_{a}^{t} y^{+}(\tau) p(\tau) \Delta \tau .
\end{aligned}
$$

By using Theorem 6.4 of [15] on (27) and (28), respectively, we obtain

$$
\begin{aligned}
& y^{-}(t) \leq f^{-}(t)+\int_{a}^{t} e_{p}(t, \sigma(\tau)) f^{-}(\tau) p(\tau) \Delta \tau, \\
& y^{+}(t) \leq f^{+}(t)+\int_{a}^{t} e_{p}(t, \sigma(\tau)) f^{+}(\tau) p(\tau) \Delta \tau .
\end{aligned}
$$

Again by using the Kulisch-Miranker order (1) on inequalities (29) and (30), respectively, we obtain

$$
\begin{aligned}
{\left[y^{-}(t), y^{+}(t)\right] \leq } & {\left[f^{-}(t)+\int_{a}^{t} e_{p}(t, \sigma(\tau)) f^{-}(\tau) p(\tau) \Delta \tau, f^{+}(t)\right.} \\
& \left.+\int_{a}^{t} e_{p}(t, \sigma(\tau)) f^{+}(\tau) p(\tau) \Delta \tau\right], \\
Y(t) \leq F(t)+ & \int_{a}^{t} e_{p}(t, \sigma(\tau)) F(\tau) p(\tau) \Delta \tau .
\end{aligned}
$$

Thus we have our claim.

Corollary 3.7 Let $Y \in C_{\mathrm{rd}}\left([a, b]_{\mathbb{T}}, \mathcal{K}_{C}\right), p \in \mathcal{R}^{+}\left([a, b]_{\mathbb{T}}, \mathbb{R}\right), p \geq 0$, and $X_{0} \in \mathcal{K}_{C}$. If

$$
Y(t) \leq X_{0}+\int_{a}^{t} Y(t) p(t) \Delta t \quad \text { for all } t \in[a, b]_{\mathbb{T}}
$$

then

$$
F(t) \leq e_{p}(t, a) X_{0} \quad \text { for all } t \in[a, b]_{\mathbb{T}}
$$

Proof By taking $F(t)=X_{0}$, in Theorem 3.6, we obtain (32).

Corollary 3.8 Let $Y \in C_{\mathrm{rd}}\left([a, b]_{\mathbb{T}}, \mathcal{K}_{C}\right), p \in \mathcal{R}^{+}\left([a, b]_{\mathbb{T}}, \mathbb{R}\right), p \geq 0$, and it satisfies the interval integral inequality

$$
Y(t) \leq \int_{a}^{t} Y(t) p(t) \Delta t \quad \text { for all } t \in[a, b]_{\mathbb{T}}
$$

then

$$
Y(t) \leq\{0\}
$$

for all $t \in[a, b]_{\mathbb{T}}$. 
Theorem 3.9 Let $F, Q, Y \in C_{\mathrm{rd}}\left([a, b]_{\mathbb{T}}, \mathcal{K}_{C}\right), p, b \in \mathcal{C}_{\mathrm{rd}}\left([a, b]_{\mathbb{T}}, \mathbb{R}\right), p, b \geq 0$, and it satisfies the interval integral inequality

$$
Y(t) \leq F(t)+b(t) \int_{a}^{t}[Y(\tau) p(\tau)+Q(t)] \Delta \tau \quad \text { for all } t \in[a, b]_{\mathbb{T}}
$$

then

$$
Y(t) \leq F(t)+b(t) \int_{a}^{t} e_{p b}(t, \sigma(\tau))(F(\tau) p(\tau)+Q(t)) \Delta \tau
$$

for all $t \in[a, b]_{\mathbb{T}}$.

Proof Let $F, Q, Y:[a, b]_{\mathbb{T}} \rightarrow \mathcal{K}_{C}$ be interval-valued functions such that $F(t)=\left[f^{-}(t), f^{+}(t)\right]$, $Q(t)=\left[q^{-}(t), q^{+}(t)\right]$ and $Y(t)=\left[y^{-}(t), y^{+}(t)\right]$. Then from inequality (33) we have

$$
\begin{aligned}
{\left[y^{-}(t), y^{+}(t)\right] \leq } & {\left[f^{-}(t)+b(t) \int_{a}^{t}\left(y^{-}(\tau) p(\tau)+q^{-}(\tau)\right) \Delta \tau,\right.} \\
& \left.f^{+}(t)+b(t) \int_{a}^{t}\left(y^{+}(\tau) p(\tau)+q^{+}(\tau)\right) \Delta \tau\right] .
\end{aligned}
$$

By using the Kulisch-Miranker order (1) we obtain

$$
\begin{aligned}
& y^{-}(t) \leq f^{-}(t)+b(t) \int_{a}^{t}\left(y^{-}(\tau) p(\tau)+q^{-}(\tau)\right) \Delta \tau, \\
& y^{+}(t) \leq f^{+}(t)+b(t) \int_{a}^{t}\left(y^{+}(\tau) p(\tau)+q^{+}(\tau)\right) \Delta \tau .
\end{aligned}
$$

Applying Theorem 3.1 of [20] on (34) and (35) we obtain

$$
\begin{aligned}
& y^{-}(t) \leq f^{-}(t)+b(t) \int_{a}^{t} e_{p b}(t, \sigma(\tau))\left(f^{-}(\tau) p(\tau)+q^{-}(\tau)\right) \Delta \tau, \\
& y^{+}(t) \leq f^{+}(t)+b(t) \int_{a}^{t} e_{p b}(t, \sigma(\tau))\left(f^{+}(\tau) p(\tau)+q^{+}(\tau)\right) \Delta \tau .
\end{aligned}
$$

From (36) and (37), using the Kulisch-Miranker order (1) yields

$$
\begin{gathered}
{\left[y^{-}(t), y^{+}(t)\right] \leq\left[f^{-}(t)+b(t) \int_{a}^{t} e_{p b}(t, \sigma(\tau))\left[f^{-}(\tau) p(\tau)+q^{-}(\tau)\right] \Delta \tau,\right.} \\
\left.f^{+}(t)+b(t) \int_{a}^{t} e_{p b}(t, \sigma(\tau))\left[f^{+}(\tau) p(\tau)+q^{+}(\tau)\right] \Delta \tau\right], \\
Y(t) \leq F(t)+b(t) \int_{a}^{t} e_{p b}(t, \sigma(\tau))(F(\tau) p(\tau)+Q(\tau)) \Delta \tau .
\end{gathered}
$$

Thus we have our claim.

The next corollary is obtained by taking $F(t)=Q(t)=\{0\}$ in Theorem 3.9. 
Corollary 3.10 Suppose $Y(t) \in C_{\mathrm{rd}}\left([a, b]_{\mathbb{T}}, \mathcal{K}_{C}\right)$ and $p, b \in \mathcal{C}_{\mathrm{rd}}\left([a, b]_{\mathbb{T}}, \mathbb{R}\right), p, b \geq 0$, and satisfies the interval integral inequality

$$
Y(t) \leq b(t) \int_{a}^{t} Y(\tau) p(\tau) \Delta \tau \quad \text { for all } t \in[a, b]_{\mathbb{T}}
$$

then

$$
Y(t) \leq\{0\}
$$

for all $t \in[a, b]_{\mathbb{T}}$.

Remark 3.11 If $b(t)=1$ in Corollary 3.10 then we obtain Corollary 3.8.

Theorem 3.12 Let $F, Q, Y \in C_{\mathrm{rd}}\left([a, b]_{\mathbb{T}}, \mathcal{K}_{C}\right), p(t), b(t) \in \mathcal{C}_{\mathrm{rd}}\left([a, b]_{\mathbb{T}}, \mathbb{R}\right), p, b \geq 0$, and it satisfies the interval integral inequality

$$
Y(t) \leq F(t)+b(t) \int_{a}^{t}\left[Y^{\sigma}(\tau) p(\tau)+Q(t)\right] \Delta \tau \quad \text { for all } t \in[a, b]_{\mathbb{T}},
$$

then

$$
Y(t) \leq F(t)+b(t) \int_{a}^{t} e_{\ominus b^{\sigma} p}(t, \tau)\left[F^{\sigma}(\tau) p(\tau)+Q(t)\right] \Delta \tau
$$

for all $t \in[a, b]_{\mathbb{T}}$.

Proof Let $F, Q, Y:[a, b]_{\mathbb{T}} \rightarrow \mathcal{K}_{C}$ be interval-valued functions such that $F(t)=\left[f^{-}(t), f^{+}(t)\right]$, $Q(t)=\left[q^{-}(t), q^{+}(t)\right]$ and $Y(t)=\left[y^{-}(t), y^{+}(t)\right]$. Then from (38) we have

$$
\begin{aligned}
{\left[y^{-}(t), y^{+}(t)\right] \leq } & {\left[f^{-}(t)+b(t) \int_{a}^{t}\left[y^{-}(\sigma(\tau)) p(\tau)+q^{-}(\tau)\right] \Delta \tau,\right.} \\
& \left.f^{+}(t)+b(t) \int_{a}^{t}\left[y^{+}(\sigma(\tau)) p(\tau)+q^{+}(\tau)\right] \Delta \tau\right] .
\end{aligned}
$$

By the Kulisch-Miranker order (1) we have

$$
\begin{aligned}
& y^{-}(t) \leq f^{-}(t)+b(t) \int_{a}^{t}\left[y^{-}(\sigma(\tau)) p(\tau)+q^{-}(\tau)\right] \Delta \tau, \\
& y^{+}(t) \leq f^{+}(t)+b(t) \int_{a}^{t}\left[y^{+}(\sigma(\tau)) p(\tau)+q^{+}(\tau)\right] \Delta \tau .
\end{aligned}
$$

Applying Theorem 3.6 of [20] on inequalities (39) and (40) we obtain

$$
\begin{aligned}
& y^{-}(t) \leq f^{-}(t)+b(t) \int_{a}^{t} e_{\ominus b^{\sigma} p}(t, \sigma(\tau))\left[f^{-}(\tau) p(\tau)+q^{-}(\tau)\right] \Delta \tau, \\
& y^{+}(t) \leq f^{+}(t)+b(t) \int_{a}^{t} e_{\ominus b^{\sigma} p}(t, \sigma(\tau))\left[f^{+}(\tau) p(\tau)+q^{+}(\tau)\right] \Delta \tau .
\end{aligned}
$$


Again using the Kulisch-Miranker order (1) on inequalities (41) and (42) we get

$$
\begin{gathered}
{\left[y^{-}(t), y^{+}(t)\right] \leq\left[f^{-}(t)+b(t) \int_{a}^{t} e_{\ominus b^{\sigma} p}(t, \sigma(\tau))\left[f^{-}(\tau) p(\tau)+q^{-}(\tau)\right] \Delta \tau,\right.} \\
\left.f^{+}(t)+b(t) \int_{a}^{t} e_{\ominus b^{\sigma} p}(t, \sigma(\tau))\left[f^{+}(\tau) p(\tau)+q^{+}(\tau)\right] \Delta \tau\right], \\
Y(t) \leq F(t)+b(t) \int_{a}^{t} e_{\ominus b^{\sigma} p}(t, \tau)\left[F^{\sigma}(\tau) p(\tau)+Q(t)\right] \Delta \tau .
\end{gathered}
$$

Thus we have our claim.

Theorem 3.13 Suppose $F, Y \in C_{\mathrm{rd}}\left([a, b]_{\mathbb{T}}, \mathcal{K}_{C}\right), F \in C_{\mathrm{rd}}^{1}\left([a, b]_{\mathbb{T}}, \mathcal{K}_{C}\right), p, b \in \mathcal{C}_{\mathrm{rd}}\left([a, b]_{\mathbb{T}}, \mathbb{R}\right)$, $p, b \geq 0$ and it satisfies the interval integral inequality

$$
Y(t) \leq F(t)+\int_{a}^{t} p(\tau) Y(\tau) \Delta \tau \quad \text { for all } t \in[a, b]_{\mathbb{T}}
$$

then

$$
Y(t) \leq e_{p}(t, a) F(a)+\int_{a}^{t} e_{p}(t, \sigma(\tau)) F^{\Delta}(\tau) \Delta \tau
$$

for all $t \in[a, b]_{\mathbb{T}}$.

Proof Let $F, Y:[a, b]_{\mathbb{T}} \rightarrow \mathcal{K}_{C}$ be interval-valued functions such that $F(t)=\left[f^{-}(t), f^{+}(t)\right]$ and $Y(t)=\left[y^{-}(t), y^{+}(t)\right]$. Then from (43) we have

$$
\left[y^{-}(t), y^{+}(t)\right] \leq\left[f^{-}(t)+\int_{a}^{t} y^{-}(\tau) p(\tau) \Delta \tau, f^{+}(t)+\int_{a}^{t}\left[y^{+}(\tau) p(\tau)\right] \Delta \tau\right] .
$$

The Kulisch-Miranker order (1) yields

$$
\begin{aligned}
& y^{-}(t) \leq f^{-}(t)+\int_{a}^{t} y^{-}(\tau) p(\tau) \Delta \tau, \\
& y^{+}(t) \leq f^{+}(t)+\int_{a}^{t} y^{+}(\tau) p(\tau) \Delta \tau .
\end{aligned}
$$

Applying Theorem 3.7 of [20] on inequalities (44) and (45), respectively, we obtain

$$
\begin{aligned}
& y^{-}(t) \leq e_{p}(t, a) f^{-}(a)+\int_{a}^{t} e_{p}(t, \sigma(\tau))\left(f^{-}\right)^{\Delta}(\tau) \Delta \tau, \\
& y^{+}(t) \leq e_{p}(t, a) f^{+}(t)+b(t) \int_{a}^{t} e_{p}(t, \sigma(\tau))\left(f^{+}\right)^{\Delta}(\tau) \Delta \tau .
\end{aligned}
$$

Again by using the Kulisch-Miranker order (1) on inequalities (46) and (47) we get

$$
\begin{aligned}
{\left[y^{-}(t), y^{+}(t)\right] \leq } & {\left[e_{p}(t, a) f^{-}(a)+\int_{a}^{t} e_{p}(t, \sigma(\tau))\left(f^{-}\right)^{\Delta}(\tau) \Delta \tau\right.} \\
& \left.e_{p}(t, a) f^{+}(t)+b(t) \int_{a}^{t} e_{p}(t, \sigma(\tau))\left(f^{+}\right)^{\Delta}(\tau) \Delta \tau\right]
\end{aligned}
$$




$$
Y(t) \leq e_{p}(t, a) F(a)+\int_{a}^{t} e_{p}(t, \sigma(\tau)) F^{\Delta}(\tau) \Delta \tau
$$

Thus we have our claim.

We need the following lemma to obtain our next result.

Lemma 3.14 (Theorem 2.5 of [20]) Let $t_{0} \in \mathbb{T}^{\kappa}$ and assume $k: \mathbb{T} \times \mathbb{T} \rightarrow \mathbb{R}$ is continuous at $(t, t)$ where $t \in \mathbb{T}^{\kappa}$ with $t>t_{0}$. Also assume that $k(t, \cdot)$ is $r d$-continuous on $\left[t_{0}, \sigma(t)\right]$. Suppose that for each $\varepsilon>0$ there exists a neighborhood $U$ of $t$, independent of $\tau \in\left[t_{0}, \sigma(t)\right]$, such that

$$
\left|k(\sigma(t), \tau)-k(s, \tau)-k^{\Delta_{t}}(t, \tau)(\sigma(t)-s)\right| \leq \varepsilon|\sigma(t)-s| \quad \text { for all } s \in U
$$

where $k^{\Delta_{t}}$ denotes the derivative of $k$ with respect to the first variable. Then

$$
g^{\Delta_{t}}(t)=\int_{t_{0}}^{t} k^{\Delta_{t}}(t, \tau) \Delta \tau+k(\sigma(t), t)
$$

where $g(t)=\int_{t_{0}}^{t} k(t, \tau) \Delta \tau$.

Theorem 3.15 Suppose $F \in C_{\mathrm{rd}}\left([a, b]_{\mathbb{T}}, \mathcal{K}_{C}^{+}\right)$and $u \in C_{\mathrm{rd}}\left([a, b]_{\mathbb{T}}, \mathbb{R}\right)$ are non-negative functions, $C$ is a non-negative constant interval. Let $k(t, s)$ be defined in Lemma 3.14 such that $k(\sigma(t), t)$ and $k^{\Delta t}(t, s)$ are non-negative and $r d$-continuous for all $s, t \in[a, b]_{\mathbb{T}}$ with $s \leq t$, and then

$$
F(t) \leq C+\int_{t_{0}}^{t} u(s)\left[F(s)+\int_{t_{0}}^{s} k(s, \tau) F(\tau) \Delta \tau\right] \Delta s \quad \text { for all } t \in[a, b]_{\mathbb{T}}
$$

implies

$$
F(t) \leq\left[1+\int_{t_{0}}^{t} u(s) e_{p}\left(s, t_{0}\right) \Delta s\right] C \text { for all } t \in[a, b]_{\mathbb{T}},
$$

where $p\left(t, t_{0}\right)=u(t)+k(\sigma(t), t)+\int_{t_{0}}^{t} k^{\Delta_{t}}(t, s) \Delta s$.

Proof Let $F \in C_{\mathrm{rd}}\left([a, b]_{\mathbb{T}}, \mathcal{K}_{C}\right)$ so $F(t)=\left[f^{-}(t), f^{+}(t)\right]$ and $C=\left[c^{-}, c^{+}\right]$. It follows from (48) that

$$
\begin{aligned}
{\left[f^{-}(t), f^{+}(t)\right] \leq } & {\left[c^{-}, c^{+}\right]+\int_{t_{0}}^{t} u(s)\left[\left[f^{-}(s), f^{+}(s)\right]\right.} \\
& \left.+\int_{t_{0}}^{s} k(s, \tau)\left[f^{-}(\tau), f^{+}(\tau)\right] \Delta \tau\right] \Delta s \\
= & {\left[c^{-}+\int_{t_{0}}^{t} u(s)\left[f^{-}(s)+\int_{t_{0}}^{s} k(s, \tau) f^{-}(\tau) \Delta \tau\right] \Delta s,\right.} \\
& \left.c^{+}+\int_{t_{0}}^{t} u(s)\left[f^{+}(s)+\int_{t_{0}}^{s} k(s, \tau) f^{+}(\tau) \Delta \tau\right] \Delta s\right] .
\end{aligned}
$$


Applying the Kulisch-Miranker order (1), we have

$$
\begin{aligned}
& f^{-}(t) \leq c^{-}+\int_{t_{0}}^{t} u(s)\left[f^{-}(s)+\int_{t_{0}}^{s} k(s, \tau) f^{-}(\tau) \Delta \tau\right] \Delta s, \\
& f^{+}(t) \leq c^{+}+\int_{t_{0}}^{t} u(s)\left[f^{+}(s)+\int_{t_{0}}^{s} k(s, \tau) f^{+}(\tau) \Delta \tau\right] \Delta s .
\end{aligned}
$$

By using Theorem 3.4 of [12] on (49), (50), respectively, we obtain

$$
\begin{aligned}
f^{-}(t) & \leq\left(1+\int_{t_{0}}^{t} u(s) e_{p}\left(s, t_{0}\right) \Delta s\right) c^{-} \\
f^{+}(t) & \leq\left(1+\int_{t_{0}}^{t} u(s) e_{p}\left(s, t_{0}\right) \Delta s\right) c^{+}
\end{aligned}
$$

So, the Kulisch-Miranker order (1) yields

$$
F(t) \leq\left[1+\int_{t_{0}}^{t} u(s) e_{p}\left(s, t_{0}\right) \Delta s\right] C \quad \text { for all } t \in[a, b]_{\mathbb{T}}
$$

where $p\left(t, t_{0}\right)=u(t)+k(\sigma(t), t)+\int_{t_{0}}^{t} k^{\Delta_{t}}(t, s) \Delta s$.

Corollary 3.16 Under the same assumption of Theorem 3.15

$$
F(t) \leq C+\int_{t_{0}}^{t} u(s)\left[F(s)+\int_{t_{0}}^{s} k(s, \tau) F(\tau) \Delta \tau\right] \Delta s \quad \text { for all } t \in[a, b]_{\mathbb{T}}
$$

implies

$$
F(t) \leq \exp \left[\int_{t_{0}}^{t} u(s) e_{p}\left(s, t_{0}\right) \Delta s\right] C \quad \text { for all } t \in[a, b]_{\mathbb{T}} .
$$

Remark 3.17 If we take $k(t, s)=0$ in Theorem 3.15 then we obtain Corollary 3.7.

Letting $k(t, s)=h(t) g(t)$ in Theorem 3.15 we obtain the following corollary.

Corollary 3.18 Suppose $h, g \in C_{\mathrm{rd}}(\mathbb{T}, \mathbb{R})$ are non-negative functions and $h^{\Delta}(t)$ exists and is non-negative and $r d$-continuous and satisfies the interval integral inequality

$$
F(t) \leq C+\int_{t_{0}}^{t} u(s)\left[F(s)+h(s) \int_{t_{0}}^{s} g(\tau) F(\tau) \Delta \tau\right] \Delta s \quad \text { for all } t \in \mathbb{T}
$$

then

$$
F(t) \leq\left[1+\int_{t_{0}}^{t} u(s) e_{p}\left(s, t_{0}\right) \Delta s\right] C
$$

for all $t \in[a, b]_{\mathbb{T}}$, where $p\left(t, t_{0}\right)=u(t)+h(\sigma(t)) g(t)+h^{\Delta}(t) \int_{t_{0}}^{t} g(s) \Delta s$.

If we take $\mathbb{T}=\mathbb{R}$ in Theorem 3.15 then we have the following corollary. 
Corollary3.19 Suppose $F \in C_{\mathrm{rd}}\left([a, b]_{\mathbb{R}}, \mathcal{K}_{C}\right)$ and $u \in C_{\mathrm{rd}}(\mathbb{R}, \mathbb{R})$ are non-negative functions, let $C \in \mathcal{K}_{C}^{+}$be a constant interval. Let $k(t, s)$ and $k^{t}(t, s)$ be non-negative and $r d$-continuous for $s, t \in[a, b]$ and if

$$
F(t) \leq C+\int_{t_{0}}^{t} u(s)\left[F(s)+\int_{t_{0}}^{s} k(s, \tau) F(\tau) d \tau\right] d s \text { for all } t \geq t_{0},
$$

then

$$
F(t) \leq\left[1+\int_{t_{0}}^{t} u(s) \exp \left(\int_{t_{0}}^{s} p\left(\tau t_{0}\right) d \tau\right) d s\right] C \text { for all } t \geq t_{0}
$$

where $p\left(t, t_{0}\right)=u(t)+k(t, t)+\int_{t_{0}}^{t} \frac{\partial}{\partial t} k(t, \sigma) d \sigma$.

The next result is another version of Theorem 3.15 obtained without the condition of differentiability of $k(t, s)$ with respect to first variable.

Theorem 3.20 Suppose $F \in C_{\mathrm{rd}}\left([a, b]_{\mathbb{T}}, \mathcal{K}_{C}\right)$ and $u \in C_{\mathrm{rd}}(\mathbb{T}, \mathbb{R})$ are non-negative functions, $C$ is a constant interval. Let $k(t, s)$ be non-negative and $r d$-continuous for $s, t \in \mathbb{T}$ with $s \leq t$ and

$$
F(t) \leq C+\int_{t_{0}}^{t} u(s)\left[F(s)+\int_{t_{0}}^{s} k(s, \tau) F(\tau) \Delta \tau\right] \Delta s \quad \text { for all } t \in[a, b]_{\mathbb{T}}
$$

then

$$
F(t) \leq e_{q}\left(t, t_{0}\right) C
$$

for all $t \in[a, b]_{\mathbb{T}_{0}}$, where $q\left(t, t_{0}\right)=u(t)\left(1+\int_{t_{0}}^{t} k(t, s) \Delta s\right)$.

Proof By using Theorem 3.13 of [12] in (49)

$$
\begin{aligned}
& f^{-}(t) \leq e_{q}\left(t, t_{0}\right) c^{-} \\
& f^{+}(t) \leq e_{q}\left(t, t_{0}\right) c^{+} .
\end{aligned}
$$

By the Kulisch-Miranker order (1), we obtain

$$
F(t) \leq e_{q}\left(t, t_{0}\right) C \text { for all } t \in[a, b]_{\mathbb{T}},
$$

where $q\left(t, t_{0}\right)=u(t)\left(1+\int_{t_{0}}^{t} k(t, s) \Delta s\right)$.

\section{Conclusions}

In this paper some results as regards Gronwall type inequalities for interval-valued functions, which provide explicit bounds on unknown functions, are presented. The results can be useful in the study of the uniqueness of the solution for interval-valued differential equations or interval-valued integro-differential equations. The results also unify and extend some continuous inequalities and some new results in the discrete case are proved. 
Competing interests

The authors declare that they have no competing interests.

\section{Authors' contributions}

All authors contributed equally to the writing of this paper. All authors read and approved the final manuscript.

\section{Acknowledgements}

The last author acknowledge the Higher Education Commission of Pakistan for its financial support.

Received: 22 January 2015 Accepted: 26 August 2015 Published online: 11 September 2015

\section{References}

1. Gronwall, T: Note on the derivatives with respect to a parameter of the solutions of a system of differential equations. Ann. Math. 20, 292-296 (1919)

2. Bellman, R: The stability of solutions of linear differential equations. Duke Math. J. 10, 643-647 (1943)

3. Bainov, D, Simeonov, P: Integral Inequalities and Applications. Kluwer Academic, Dordrecht (1992)

4. Mitrinovic, DS, Pecaric, JE, Fink, AM: Inequalities Involving Functions and Their Integrals and Derivatives. Kluwer Academic, Dordrecht (1991)

5. Pachpatte, BG: On note on Gronwall-Bellman inequality. J. Math. Anal. Appl. 44, 758-762 (1973)

6. Pachpatte, BG: On some retarded integral inequalities and applications. J. Inequal. Pure Appl. Math. 3(2), 1-7 (2002)

7. Moore, RE: Interval Analysis. Prentice Hall, Englewood Cliffs (1966)

8. Chalco-Cano, Y, Flores-Franulic, A, Román-Flores, H: Ostrowski type inequalities for interval-valued functions using generalized Hukuhara derivative. Comput. Appl. Math. 31, 457-472 (2012)

9. Hilger, S: Ein Maßkettenkalkül mit Anwendung auf Zentrumsmannigfaltigkeiten. PhD thesis, Universität Würzburg (1988)

10. Lupulescu, $\mathrm{V}$ : Hukuhara differentiability of interval-valued functions and interval differential equations on time scales. Inf. Sci. 248, 50-67 (2013)

11. Markov, S: Calculus for interval functions of a real variables. Computing 22, 325-337 (1979)

12. Choi, SK, Koo, N: On a Gronwall type inequalities on time scales. J. Chungcheong Math. Soc. 21, 137-147 (2010)

13. Li, WN, Sheng, W: Some Gronwall type inequalities on time scales. J. Math. Inequal. 4(1), 67-76 (2010)

14. Román-Flores, H, Chalco-Cano, Y, Silva, GN: A note on Gronwall type inequality for interval valued functions. In: IFSA World Congress and NAFIPS Annual Meeting (IFSA/NAFIPS) (2013)

15. Bohner, M, Peterson, A: Dynamic Equations on Time Scales: An Introduction with Applications. Birkhäuser, Basel (2001)

16. Kulish, UW, Miranker, WL: Computer Arithmetic in Theory and Practice. Academic Press, New York (1982)

17. Stefanini, L: A generalization of Hukuhara difference and division for interval and fuzzy arithmetic. Fuzzy Sets Syst. $161,1564-1584(2010)$

18. Chalco-Cano, Y, Román-Flores, H, Jiménez-Gamero, MD: Generalized derivative and $\pi$-derivative for set-valued functions. Inf. Sci. 181, 2177-2188 (2011)

19. Malinowski, MT: Interval Cauchy problem with a second type Hukuhara derivative. Inf. Sci. 213, $94-105$ (2012)

20. Akin-Bohner, E, Bohner, M, Akin, F: Pachpatte inequalities on time scales. J. Inequal. Pure Appl. Math. 6(1), Article 6 (2005)

\section{Submit your manuscript to a SpringerOpen ${ }^{\ominus}$ journal and benefit from:}

- Convenient online submission

Rigorous peer review

- Immediate publication on acceptance

- Open access: articles freely available online

- High visibility within the field

- Retaining the copyright to your article 